\title{
Fusion of Biplane Fluoroscopy with Fiber Bragg Grating for 3D Catheter Shape Reconstruction
}

\author{
M. Ourak ${ }^{1}$, S. De Buck ${ }^{2,3}$, X.T. Ha ${ }^{1}$, O. Al-Ahmad ${ }^{1,4}$, K. Bamps ${ }^{2}$, J. Ector ${ }^{2}$ and E. Vander Poorten ${ }^{1}$
}

\begin{abstract}
Nowadays, navigating therapeutic catheters takes place under 2D fluoroscopic imaging. This requires considerable training of the clinician and exposes him/her to X-ray radiation. Researchers have increasingly investigated alternative sensing techniques. In this respect, Fiber Bragg Grating (FBG)-based shape sensing is gaining popularity. This paper proposes two approaches to fuse FBG with fluoroscropy, improve the understanding of the 3-dimensional shape while reducing fluoroscopy use. This paper proposes two FBG-fluoroscopy fusion approaches that combine fluoroscopy and FBG measurements. A comparison is performed between 3D shape reconstructions based on biplane fluoroscopy, rigidly fused multi-core FBG and dynamically fused FBG shape reconstruction. To verify the performance of the different approaches experiments were performed with custom made catheter in a CathLab on 3D printed tubes with known ground truth shape. The experiments showed overall acceptable errors for the targeted application with a maximum below $2 \mathrm{~mm}$. The error of shape reconstruction through biplane fluoroscopy, rigid and dynamic fusion were found to be $1.51 \pm 0.04 \mathbf{m m}$, $1.77 \pm 0.29 \mathrm{~mm}$ and $1.47 \pm 0.15 \mathrm{~mm}$, respectively. Thus, FBGfluoroscopy fusion offers comparable results to fluoroscopy and may substantially reduce the radiation dose through optimal acquisition frequency.
\end{abstract}

Index Terms-steerable catheters, surgical robotics, sensor fusion, performance evaluation and benchmarking

\section{INTRODUCTION}

Catheters are essential and versatile instruments that enable minimally invasive treatment of the heart. Thanks to this technology access trauma in the cardiothoracic anatomy can be decreased and risks for intra-operative and post-procedural complications and co-morbidity can be reduced [1]. Previously, catheters merely served as flexible tubes that were used to monitor patients or deliver drugs [2]. Yet, currently, these devices carry sophisticated implants, sensors or offer operative functionality. Catheters are broadly employed for coronary stenting, vascular repair, repair of congenital heart defects and valve surgery or for treating cardiac rhythm disorders like atrial fibrillation by radio-frequency ablation [3]. In this work, we focus on the latter application. The aim is to track the catheter shape in real-time to provide trajectory for guiding, monitoring or robotically steered ablation.

\footnotetext{
${ }^{1}$ Mouloud Ourak, Xuan Thao Ha, Omar Al-Ahmad, and Emmanuel Vander Poorten are with Department of Mechanical Engineering, KU Leuven, Belgium

${ }^{2}$ Stijn De Buck, Kobe Bamps, Joris Ector are with Department of Cardiology, University Hospital Gasthuisberg, University of Leuven, Belgium

${ }^{3}$ Stijn De Buck is with Departments of Imaging and pathology and ESAT, KU Leuven, Belgium

${ }^{4}$ Omar Al-Ahmad, is with FBGS International NV, Geel, Belgium firstname. lastnamedkuleuven. be
}

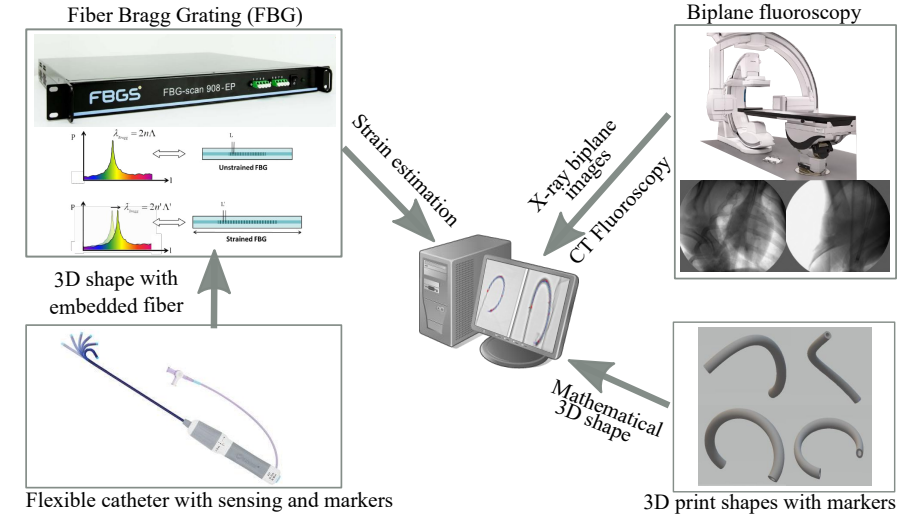

Fig. 1. Experimental platform for 3D catheter shape reconstruction featuring FBG interrogation for acquisition of catheter strain, biplane fluoroscopy for $\mathrm{X}$-ray-based shape reconstruction, 3D printed hollow tubular shapes following mathematical functions serving as ground-truth and a flexible sensorized catheter with integrated multi-core FBG optical fiber (FBGS Int., Geel, Belgium) and radio-opaque markers.

Prior research has investigated different approaches to improve the understanding of the catheter shape inside the patient. The developed approaches can be roughly classified into: 1) model-based, 2) sensor-based, and 3) methods that combine both. The first group only uses knowledge of the insertion depth and then relies on a kinematic model of the catheter and its environment to estimate the catheter configuration [4], [5]. This requires an accurate and complex modeling procedure calling for highly intensive computations complicating realtime use. Furthermore, the models are closely linked to each specific instrument and patients which limits their broad use.

The second group relies on intra-operative sensing to reconstruct the shape. Different sensing modalities have been investigated in the past. Nowadays, fluoroscopy is very much established in clinical practice for guiding catheter navigation. However, fluoroscopy only provides $2 \mathrm{D}$ visualization. The interventionist is asked to produce a mental construct that correlates a 3D representation in her/his mind with the 2D projective view form the fluoroscope. Wagner et al. proposed to simplify this mental task by mathematically reconstructing the 3D shape from 2D images taken under two different angles. This is known as biplane fluoroscopy. Nevertheless, biplane fluoroscope worsens x-ray radiation exposure even more [6].

Other approaches rely on sensors that are embedded inside the catheter's body that provide so-called proprioceptive sensing capabilities [7], [8]. Electromagnetic tracking (EMT) is well-established [9], but recently, optic shape sensing for example by means of Fiber Bragg Gratings (FBGs) is gaining in popularity due to attractive properties such as miniature 
dimensions, immunity to electromagnetic disturbances, fast interrogation rates, suitability for distributed sensing (or multiplexing), simple integration within systems and high mechanical strength [10]. These properties allow the FBG sensors to cover a range of clinical uses: accurate estimation of the catheter 3D shape, detection of the force interaction with the external environment at the tip or along the catheter [11] and temperature measurement in real time [12]. Two main technologies exist for fiber strain sensing: Fiber Bragg Grating (FBG) and Rayleigh scattering. Rayleigh scattering based techniques have near-continuous spatial strain sensing resolution thanks to scattering that occurs throughout the fiber length. On the other hand FBG-based strain sensing relies on reflected wavelengths from an emitted light spectrum. The following factors somewhat limit the adoption of fiber deformation detection techniques: sensor twist issues where it is not easy to distinguish between twist- or bend-induced fiber deformation, imperfect knowledge of the exact location of the sensor due to variability in manufacturing and assembly processes, and the accumulation of estimation errors due to the integration of curvature and bend angle errors when calculating the 3D shape along the entire length of the fiber. The resulting integration errors can cause quite significant deviations [13]. The work in this paper focuses on FBG fibers and more specifically on straight multi-core fibers (MCFs) [14] to reconstruct the shape. Aside to MCFs, helically wrapped fibers are also being developed to improve the quality of shape reconstruction and reduce twist. However, this method requires more elaborated catheter integration methods [15].

The paper presents a first evaluation study between shape reconstruction based upon biplane fluoroscopy and FBGs, the latter progressing earlier work by Al-Ahmad et al. in [10]. The work proposes a new registration method that projects the different reconstructed shapes in the same, here biplane fluoroscopic, coordinate frame for data-fusion in an absolute frame of reference. The position of markers under fluoroscopy is discussed and analyzed. Finally, a method to dynamically fuse both sensor modalities based on a probabilistic framework that build upon the work from $\mathrm{Ha}$ et al. [16] is modified and applied. It is shown that fusion between fluoroscopy and FBGs is possible and allows simplifying the complexity of shapesensing catheters compared to the earlier versions by Ha et al. where both EMT and FBGs had to be integrated [16]. To the knowledge of the authors, this is the first study in which biplane fluoroscopy and FBG sensing have been fused and compared experimentally. Furthermore, the paper provides a comparison of the different shape reconstruction techniques in an absolute reference frame thereby avoiding unmeasurable offsets along the fiber path that would otherwise have to be registered with Iterative Closest Point (ICP).

The paper is organized as follows: Section II describes the experimental setup used during the evaluation. Section III details the reconstruction methods that were developed for each modality. An experimental validation applied on 4 different shapes is presented in Section IV. Finally, a summary of the results with some concluding remarks and directions of future work is given in Section V.

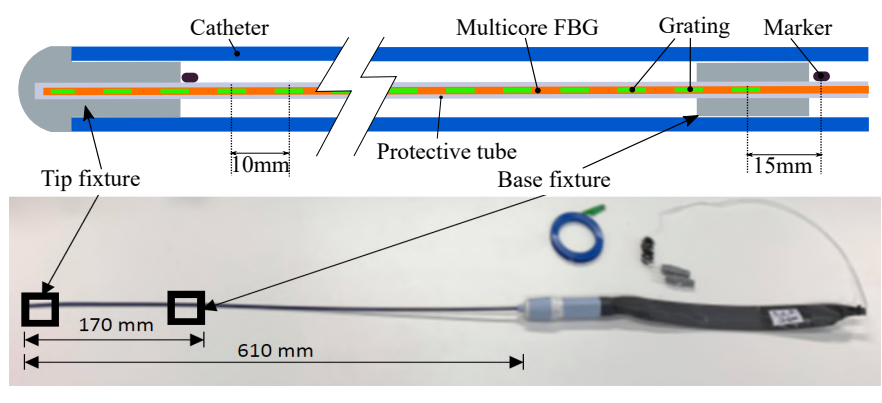

Fig. 2. Schematic of the prototype catheter that was employed in the experiments. Fitted with a multi-core Fiber Bragg Grating (MCF) optical fiber in its central channel, the Destino catheter sheath (Oscor, Düsseldorf, Germany) is further equipped with 4 radio-opaque markers that are visible under fluoroscopy and support sensor fusion of FBG and fluoroscopy.

\section{EXPERIMENT SETUP}

To evaluate the performance of the 3 different shape reconstruction methods and compare to a ground-truth shape, the experimental setup shown in Figure 1 was conceived and installed in the CathLab. The following assemblies formed part of this experimental setup.

A steerable bidirectional 8.5 Fr Destino sheath (Oscor, Düsseldorf, Germany), shown in Figure 1, was modified to allow embedding a multi-core FBG fiber. Markers are placed in the protective tube of the FBG fiber at $175 \mu \mathrm{m}$ from the fiber surface. The distance is small and not accounted for at this stage.

Two 3D print fixtures (VERO WHITE RGD835 by stratasys) were fitted inside the sheath, one at the tip and the other at a distance of approximately $170 \mathrm{~mm}$ from the tip, to hold the fiber in the center. The two fixtures are glued to the protective tube of the fiber to make sure that the two fixtures remain at the correct distances. Finally, markers, visible under fluoroscopy, are fixed both on the protective tube of the MCF fibers in order to have uniquely identifiable points that facilitate fluoroscopy based reconstruction. Also the 3D-printed tubes (TuskXC2700T) were equipped with such markers in order to relate the ground truth $3 \mathrm{D}$ shape to the individual reconstruction experiments. As explained in the following a number of measurement setups were constructed to run and verify the 3 catheter shape reconstruction methods.

A first setup was used in order to reconstruct the catheter shape solely relying on biplane fluoroscopy (Siemens Artis Biplane, Siemens Healthineers, Forcheim, Germany). Hereto, the fluoroscopic setup was first calibrated. Using the calibration and after localising their projection in both images, we determined the $3 \mathrm{D}$ position of the radio-opaque markers attached to the catheter. The orthogonal position of the two fluoroscopy $\mathrm{C}$-arms helped minimizing the uncertainty on the reconstruction in this biplane setup. The derived catheter shape is provided in an absolute coordinate system $O_{f}$ fixed relative to the fluoroscopy devices and is consequently external to the catheter.

A second setup was prepared to perform shape reconstruction through the integrated FBG optical fiber. A $250 \mu \mathrm{m}$ four-core fiber consisting of 68 FBGs was integrated in the 8.5 Fr Destino catheter sheath. Each core of the multi-core 
fiber contains 17 gratings distributed over a length of $170 \mathrm{~mm}$ at $10 \mathrm{~mm}$ separation between each grating. The length of each grating is $4 \mathrm{~mm}$. Each grating features a different central wavelength such that the associated strain can be associated easily. An interrogator (FBG-Scan 804, FBGS, Belgium) polled the $68(4 \times 17)$ gratings at a frequency of $100 \mathrm{~Hz}$. The shape reconstructed from the FBG is computed in coordinate system relative to the catheter and reconstructed at $60 \mathrm{~Hz}$. To transform it to the absolute coordinate system $O_{f}$, a rigid registration procedure based on radio-opaque markers of the catheter is adopted. All the experiments are carried out on a computer with processor Intel $2.1 \mathrm{GHz}$ core i7 and $32 \mathrm{~GB}$ of RAM, running Ubuntu 16.04.

A third shape reconstruction method employs a dynamic fusion of the MCF with biplane fluoroscopy. The model strain information from the FBGs projected on the two biplane fluoroscopy is fused with the segmented shape of the catheter on the two biplane images. The proposed approach mitigates the intrinsic problems of FBG-based shape reconstruction (twist sensitivity) and fluoroscopy-based reconstruction (i.e. radiation) by iteratively estimating and updating the parameters of the MCF model. The shape that is reconstructed by integrating the FBG model is shown to be more accurate, but is also more robust and induces less exposure to harmful radiation in the case of an ablation procedure where fluoroscopy is the only detection method used or in support of 3D electroanatomic mapping. Similar to the reconstruction relying only on FBG sensing, the shape is obtained in a coordinate frame relative to the catheter body. This again calls for a registration step in Section III-B2 to transform the shape to the absolute coordinate frame $O_{f}$.

The ground-truth shape is derived from the CBCT fluoroscopy centerline segmented from the 3D-printed hollow geometric shapes. The four 3D printed shapes were designed following mathematical functions with constant and variable curvatures between $0,00969 \mathrm{~mm}^{-1}$ and $0.035 \mathrm{~mm}^{-1}$. Four radio-opaque beads were attached also to the $3 \mathrm{D}$ printed model in order to express the ground-truth relative to the absolute frame $O_{f}$. The details of the different reconstruction and registration methods are presented next.

\section{3D SHAPE RECONSTRUCTION METHODS}

\section{A. Biplane fluoroscopy based Shape reconstruction}

The reconstruction of the catheter shape through fluoroscopy is composed out of four steps: fluoroscope calibration, marker detection, marker reconstruction and shape interpolation. Since in many catheter interventions the focal distance is quite large with respect to the extent of common objects like catheters and these objects of interest are centered in the field of view, we can approximate the fluoroscopy projection by a weak perspective model. Hereby the projection is modeled by following equation:

$$
\begin{aligned}
& p_{x}^{(c)}=s_{x} P_{x}^{(c)} \\
& p_{y}^{(c)}=s_{y} P_{y}^{(c)}
\end{aligned}
$$

in which $p^{(c)}$ is the image projection, $s_{x}, s_{y}$ are 2 scale factors and $P^{(c)}$ is the 3D point in the camera coordinate system. The latter is computed by transforming the world point $P^{(w)}$ to the camera coordinate system using a rigid transformation $T_{w 2 c}$.

1) Fluoroscope calibration: in order to calibrate the fluoroscope model, several biplane images of a rectangular radioopaque grid (see Figure 3) were grabbed. The grid was repositioned repeatedly while keeping the fluoroscopes fixed. Next, the wire intersection coordinates were semi-automatically determined in both images and related to each other allowing 3D grid coordinates to be assigned. Subsequently, the method proposed by De Buck et al. [17] was used to obtain an initial parameter set for every pair of calibration images. Since the fluoroscopes did not move in between calibration pairs, the motion of the calibration grid could be determined from this initialization. Next, an overall optimization was performed by minimizing the reprojection error:

$$
\underset{\eta}{\arg \min } \sum_{i=1, \ldots, n, j=1, \ldots, n}\left\|\tilde{p}_{i, j}-p_{i, j}\right\|
$$

with $\tilde{p}_{i}$ the estimated location in the images based upon projection parameters $\eta$ and $p_{i, j}$ the projection of grid point $i$ onto the image $j . \eta$ is composed of the parameters $s_{x}, s_{y}$ and $T_{w 2 c, j}$.

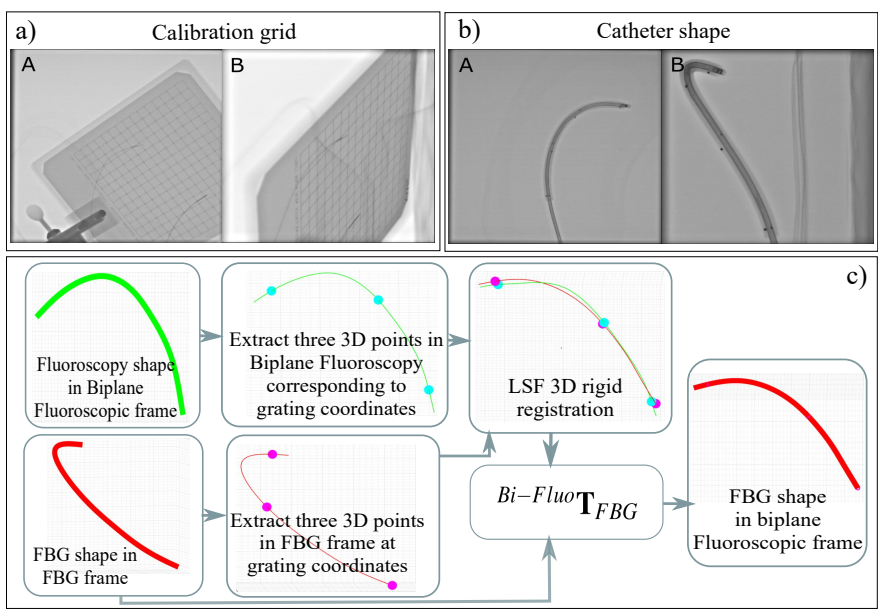

Fig. 3. View of two orthogonal fluoroscopy projections, as used in the experiments: a) the calibration grid images, b) the catheter shape images (left (A) vs. right (B)) and c)overall flowchart of the rigid registration method of FBG on the absolute coordinate frame $O_{f}$.

2) Catheter detection \& reconstruction: The radio-opaque markers (1 $\mathrm{mm}$ sphere of electric solder) integrated in the catheter were used to reconstruct the catheter shape. In this work, the 4 embedded markers were manually identified but due to the high contrast appearance of the markers, as can be seen on Figure 3(b), automated approaches (e.g. [18], [19]) could be employed equally well. Using the calibrated camera model, the corresponding $3 \mathrm{D}$ point could be estimated as the center of the normal between the pairs of lines that are backprojected from the individual images.

3) Catheter interpolation: To obtain a smooth representation of the catheter, a cubic B-spline interpolation was made. To stabilize the behaviour at the edge and when there were stronger bends in the catheter, we added additional points on the tangent outside the edge points and half way in between 
each marker. After marking in one view, the epipolar line was shown on the other in order to constrain marking the corresponding point. Hence interpolation was performed on 9 points but only the section between marker 1 to 4 was kept for further analysis.

\section{B. Shape Reconstruction by rigid fusion of Fiber Bragg Grat- ing and Fluoroscopy}

1) FBG based shape reconstruction: FBG shape reconstruction is based on strain sensing. FBGs detect a variation of strain based on the change of periodicity and refractive index of the grating. The Bragg wavelength $\lambda_{B}$ is the wavelength of the light that is reflected back from the grating. In case of MCFs, the fibers contain a central core that coincides with the fiber's neutral axis, in addition to a number of symmetrically positioned outer cores. This configuration allows compensate for disturbances induced by axial loading and/or temperature variations [10]. Thus the change in strain $\Delta \varepsilon$ with respect to the unstrained state is:

$$
\Delta \varepsilon_{i}=\frac{\Delta \lambda_{B, i}}{S_{\varepsilon} \lambda_{B_{0}, i}}-\frac{\Delta \lambda_{B, 1}}{S_{\varepsilon} \lambda_{B_{0}, 1}}
$$

where $\Delta \lambda_{B, i}$ is the wavelength shift in the outer core FBGs, $S_{\varepsilon}$ is the strain sensitivity coefficient, $\lambda_{B_{0}, i}$ is the FBG's unstrained Bragg wavelength in the outer cores, $\Delta \lambda_{B, 1}$ is the wavelength shift in the central core and $\lambda_{B_{0}, 1}$ is the grating's unstrained Bragg wavelength in the central core.

The relationship between the strain in each core $\varepsilon_{i}$ with the corresponding curvature $\kappa$ and bending plane angle $\theta_{b}$ can be obtained from the geometry as follows:

$$
\varepsilon_{i}=-\kappa d \sin \left(\theta_{b}-\frac{3 \pi}{2}-\theta_{i}\right)
$$

where $d$ is the distance between the outer cores and the central core assuming an axi-symmetric configuration, and $\theta_{i}$ is the angle of the $i^{t h}$ core with respect to a fixed axis in a crosssection of the catheter which, without loss of generality is referred here as the x-axis. As can be seen from (5), the two unknowns are curvature $\kappa$ and bend angle $\theta_{b}$. Hence in principle only two outer core strain measurements would suffice to derive these. As shown by Moore et al. [20], a closed-form solution can be obtained for the curvature $\kappa$ and the bend angle $\theta_{b}$ by defining an apparent curvature vector $\kappa_{\text {app }}$ :

$$
\begin{gathered}
\kappa_{a p p}=-\sum_{i=1}^{N} \frac{\varepsilon_{i}}{r} \cos \theta_{i} \hat{\mathbf{i}}-\sum_{i=1}^{N} \frac{\varepsilon_{i}}{r} \cos \theta_{i} \hat{\mathbf{j}} \\
\kappa=\frac{2\left|\kappa_{a p p}\right|}{N} \\
\theta_{b}=\angle \kappa_{a p p}
\end{gathered}
$$

where $\hat{\mathbf{i}}$ and $\hat{\mathbf{j}}$ are the unit vectors along the $\mathrm{x}$ - and $\mathrm{y}$-axes respectively, $N$ is the number of outer cores, and (7) represents the vector angle. The bend angle $\theta_{b}$ in (8) is obtained using the $\operatorname{atan} 2($.$) function that correctly recognizes the geometric$ quadrant of the curvature vector and limits the angle to the bounded interval $[-\pi, \pi]$.

A continuous and differentiable space curve can then be derived by using the curvature $\kappa(s)$ and torsion $\tau(s)$ profiles over the arc length $s$. Here, the torsion $\tau(s)$ is defined as the rate of change of bending angle per variation in arc length $\frac{d \theta_{b}}{d s}$. $\kappa(s)$ and $\tau(s)$ define the tangent $\mathbf{T}$, normal $\mathbf{N}$, and binormal $\mathbf{B}$ unit vectors of the curve along $s$ using the differential FrenetSerret formula [21]. Alternatively, a Bishop frame with unit vectors T, M1, and M2 can also be similarly defined. In this paper, Bishop frames [22] are used as they show improved performance compared to other methods [23]. Moreover, a plurality of other factors affect the reconstructed shape accuracy. Accordingly, the FBG-based shape reconstruction process is followed based on the recommendations in [10]. The strains $\varepsilon_{i}$ can be used to correctly reconstruct the FBG shape, but only provided that there is no twist applied on the FBG. In reality, there may a strain $\varepsilon_{t w i s t}$ that is induced by such twist. As will be explained in Section III-C our dynamic fusion is directed at estimating this twist-induced strain component and at correcting for it as follows:

$$
\varepsilon_{\text {new }, i \in\{1,2,3\}}=\varepsilon_{i}-\varepsilon_{\text {twist }} .
$$

2) Registration of FBG shape to fluoroscopy: since the FBG shape is represented in a relative reference frame, practical use for surgical guidance is limited. Hereto, we propose to employ sparse fluoroscopic measurements to register the FBG shape to the absolute reference frame $O_{f}$ of the fluoroscope. To keep the registration process lightweight, the number of markers that are used for this registration is kept at 3. This simplifies marker placement and speeds up registration. A brief analysis of the effect of varying spacing and position of markers is conducted in Section IV-A. For this work and to generate different combinations, the markers are considered to be the grating coordinates available in the two modalities.

Figure 3 (c) illustrates the registration method. To easily identify the gratings in FBG and fluoroscopy, one radioopaque marker was placed $15 \mathrm{~mm}$ before the first FBG grating of the fiber. The registration is conducted by establishing the correspondence in the fluoroscopy- and FBGbased shape between the selected markers (i.e., grating) with defined interval distances. Once these points were retrieved in both modalities a least squares fitting (LSF) registration method [24] is used to register these markers via a point-topoint 3D rigid registration. This approach relies on a singular value decomposition (SVD) and identifies the translation and rotation between frames based on a minimal amount of (noncollinear) 3D points.

\section{Dynamic fusion of FBG and fluoroscopy}

A major challenge so far in using FBGs for shape reconstruction is the limitation to distinguish the strain induced by twist from the strain induced by bending [13]. Sensor fusion is one possible option to overcome this issue. This can be achieved once the shape from the FBG is represented in the fluoroscopy frame, as presented in the previous section. The fusion method proposed in this paper works further on the work presented in [16] where FBGs were fused with EMT. Unlike the work in [16] where the 3D reconstructed shapes are represented in the electromagnetic (EM) sensor reference frame, in the proposed method we incorporated markers that 
are visible under fluoroscopy and use a reference coordinate frame attached to fluoroscopy. This reduces possible non-linear errors related to EM sensors, simplifies the correspondence (i.e., no registration is needed for the EM sensor in the catheter) and reduces the cost of additional hardware in a clinical scenario. The new framework foresees hereto a joint optimization of a 2D B-spline catheter model in the fluoroscopic image plane with the transformed MCF-based shape model. The different steps of the proposed approach are elaborated in the following.

The 3D and 2D catheter shapes are related through a projection function that is derived from the calibration of the biplane fluoroscopy. First, the projected FBG shape is derived by projecting a set of knot points $\mathbf{K}_{i}$ that are sampled from the shape estimated from FBG (as outlined in SubSec.III-B) onto the 2D image plane of the fluoroscopes. The corresponding control points can then be interpolated to obtain a B-spline representation of the shape projected in the image plane. Second, the catheter shape, captured by the fluoroscope and approximated as a polynomial (along the lines of SubSec.III-A) can be seen as a contour. Thus, a B-spline tube model $\mathbf{c}_{i}(t)$ is defined from the center line model to track the catheter shape in the $2 \mathrm{D}$ image plane

$$
\mathbf{c}_{i \in\{1, \ldots, n\}}(t)=\mathbf{b}_{i}(t) \pm r\left[\begin{array}{cc}
0 & 1 \\
-1 & 0
\end{array}\right] \hat{\mathbf{W}}_{i}(t)
$$

where $n$ is the number of knot points sampled from the estimated FBG shape, $\mathbf{b}_{i}(t)$ is the local cubic B-spline, $r$ is the radius of the catheter, $\hat{\mathbf{W}}_{i}=\frac{\mathbf{W}_{i}}{\left\|\mathbf{W}_{i}\right\|}$, with $\mathbf{W}_{i}=\frac{\delta b_{i}}{\delta t}$, is the tangent vector at the point $\mathbf{b}_{i}(t)$.

The contour $\mathbf{c}=\left\{\mathbf{c}_{1}, \ldots, \mathbf{c}_{n}\right\}$ of the $2 \mathrm{D}$ projected catheter shape in the fluoroscopic image defines two non-parametric foreground $P\left(\mathbf{y} \mid M_{f}\right)$ and background $P\left(\mathbf{y} \mid M_{b}\right)$ distributions corresponding to the foreground $\Omega_{f}$ and background $\Omega_{b}$ regions, respectively. $\mathbf{y}$ represents the pixel values at pixel position $\mathbf{x} . M=\left\{M_{f}, M_{b}\right\}$ is a model parameter that indicates either foreground or background. $P\left(\mathbf{y} \mid M_{f}\right)$ is associated with the pixels that are within the $2 \mathrm{D}$ catheter shape, while $P\left(\mathbf{y} \mid M_{b}\right)$ represents the remaining pixels.

The pixel-wise posteriors $P(\mathbf{c} \mid \Omega)$ is the probability of the catheter contour $\mathbf{c}$ given all pixel data $\Omega$. It is expressed as

$$
P(\mathbf{c} \mid \Omega)=\prod_{j=1}^{N}\left[\sum_{i=f, b}\left\{P\left(\mathbf{x} \mid \mathbf{c}, M_{i}\right) P\left(M_{i} \mid \mathbf{y}\right)\right\}\right] P(\mathbf{c})
$$

where $P(\mathbf{c} \mid \Omega)$ considers every pixel in the foreground and the background. An advantage of the proposed approach is that it allows modeling the likelihood of the foreground and background by a non-parametric probability distribution e.g. by using a histogram. Several other ways can be envisioned to encode prior knowledge. Here, gray scale values and the magnitude of a Frangi filter that is applied on the image is considered. The Frangi filter examines the multi-scale second order local structure of the image and has been employed e.g. to enhance vessels in images, which is very similar to the structures we are dealing with here [25].
The tracking of the contour $\mathbf{c}$ with respect to the knot-points is performed by maximizing the equation (12).

$$
\dot{\mathbf{c}}=\arg \min _{c} \sum_{i=1}^{N} \log \left(P\left(\mathbf{x}_{i} \mid \mathbf{c}, \mathbf{y}_{i}\right)\right)+\log (P(c))
$$

where $P\left(\mathbf{x}_{i} \mid \mathbf{c}\right)$ is defined as:

$$
\frac{H_{\mathcal{E}}\left(\Phi\left(\mathbf{x}_{i}\right)\right) P\left(\mathbf{y}_{i} \mid M_{f}\right)+\left(1-H_{\mathcal{E}}\left(\Phi\left(\mathbf{x}_{i}\right)\right)\right) P\left(\mathbf{y}_{i} \mid M_{b}\right)}{\sum_{i=1} H_{\mathcal{E}}\left(\Phi\left(\mathbf{x}_{i}\right)\right) P\left(\mathbf{y}_{i} \mid M_{f}\right)+\sum_{i=1}^{N}\left(1-H_{\mathcal{E}}\left(\Phi\left(\mathbf{x}_{i}\right)\right)\right) P\left(\mathbf{y}_{i} \mid M_{b}\right)}
$$

$\Phi(\mathbf{x})$ is the signed distance and $H_{\varepsilon}$ is a smooth Heaviside function. As suggested by [26], the Gauss-Newton algorithm is used to optimize (12).

The twist can now be estimated by fusing the initial shape reconstructed by the FBG in $O_{f}$ (see Section III-B2) and the previously correct projection in the 2D image. An optimization problem is hereto formulated to vary the dynamic twist $\varepsilon_{t w i s t}$ to make the $2 \mathrm{D}$ projected shape from the knot points matches the shape from the catheter tracking in the image plane. The value of $\varepsilon_{t w i s t}$ is now estimated by minimizing the distance $D$ between the catheter shape in the image plane calculated from the catheter tracking in 2D image step and the projection of the $3 \mathrm{D}$ shape that is reconstructed from the newly calculated strains from (9).

\section{Comparison to ground-truth}

The different 3D shapes (from ground truth (GT), fluoroscopy, FBG and fusion) need to be represented in the same reference frame in order to be evaluated. In this paper, all shapes are analysed after registration/projection into the fluoroscopic frame. The registration uses common features in the different modalities to compute the relative transformations. Radio-opaque markers are placed not only inside the catheter, but also in the surface of the four 3D-printed rigid tubes that were designed for verifying the shape estimation quality.

The ground truth shape is reconstructed as follow: 1) define the curvature and bending angle of the desired center line shape; 2) generate the corresponding 3D printed tube. Foresee in the design a minimal tolerance between the catheter and the 3D printed tube to keep play-induced errors small. Foresee locations for putting fluoroscopic markers (4 markers per shape) along the tube; 3 ) reconstruct the GT shape using cone beam computed tomography (CBCT) of the 3D printed tube alongside with the markers; 4) segment the CBCT images to extract the outer layer of the shape; 5) extract the centerline of the outer layer and the 3D coordinates of the center of the radio-opaque markers attached to the surface of the $3 \mathrm{D}$ printed tube. In order to use the GT shape with different configurations (change in position and orientation of the shape under biplane fluoroscopy), the GT is registered with the new biplane fluoroscopy using the LSF method using the markers of the outer tube and the coordinates reconstructed using biplane fluoroscopy. Figure 4 summarizes this procedure.

\section{EXPERIMENTAL VALIDATION}

The proposed approaches are validated using the experimental setup depicted in Figure 5 using 4 different rigid 3D 


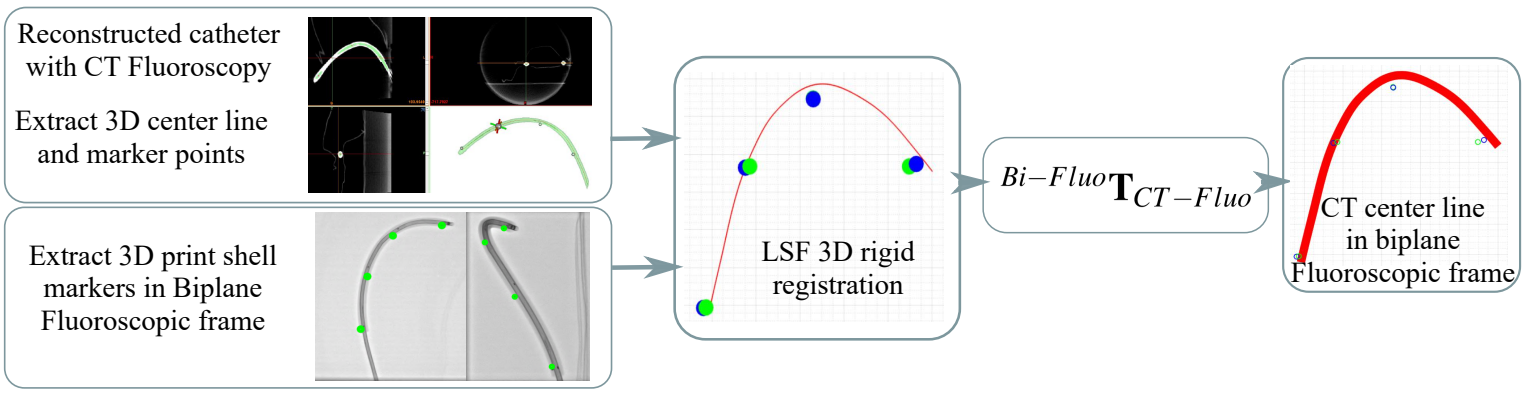

Fig. 4. An overall flowchart of the registration method to project the 3D print shape (ground truth) on the absolute coordinate frame $O_{f}$.

print shapes (see Figure 1). Per shape 4 tests are conducted whereby each time the 3D-printed tube is placed at a different position and orientation.
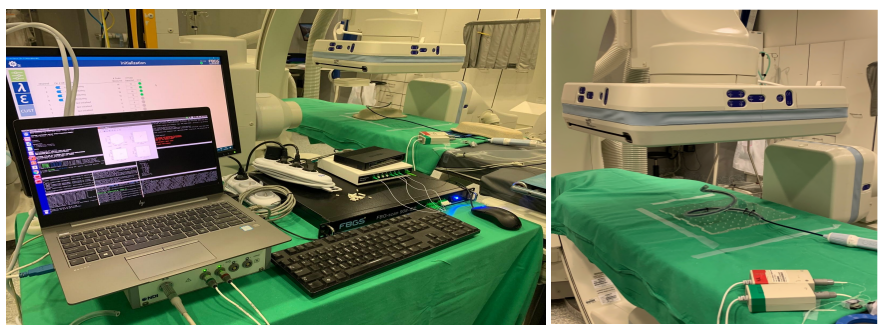

Fig. 5. Overview of the experimental platform taken in the CathLab during the experiments.

To evaluate the proposed approaches, two experiments were carried out in the CathLab. The first experiment explores the impact of the markers on the registration of the FBG shape with the fluoroscopic shape. The second set of experiments entails a comparative study between the different reconstructed shapes. During the experiments, the wavelengths of the fiber and the images from the biplane fluoroscopy were recorded. Furthermore, at the end of the experiments, biplane images of a checkerboard are grabbed to calibrate the sensors. Finally, CBCT is saved to reconstruct the 3D shape of the catheter.

\section{A. Evaluation of optimal marker distribution along catheter}

As indicated in Section III-B2, this task compares the different possible marker positions to optimally register the FBG shape to the biplane fluoroscopic frame. In this test, an incremental step of $10 \mathrm{~mm}$ corresponding to the space between grating is applied. The test covers the entire sensitive length of the fiber, i.e., over a span up to $150 \mathrm{~mm}$.

This experiment demonstrates that it is feasible to register the FBG shape onto the fluoroscopic shape with only 3 markers. By placing the markers further separated, ideally covering the full catheter length. Figure 7 shows the smallest error down to $1.77 \mathrm{~mm}$ for markers that are placed at the base, middle, and tip of the fiber. It was decided to adopt a $70 \mathrm{~mm}$ spacing between markers for the next experiments.

\section{B. Comparative study between the three catheter shape recon- struction methods}

This experiment evaluates the performance of the 3 proposed methods for 3D shape reconstruction. All shapes are registered relative to the same fluoroscopic frame. Figure 6(a) depicts the 3D curves of the 4 different shapes for each of the 4 shapes. The displayed test is representative (yet randomly selected) out of the 4 tests that were done for each shape. The error between the 4 reconstructed shapes and the ground truth shape is displayed in Figure 6(d). Finally, Figure 6(b) and 6(c) show the projected $3 \mathrm{D}$ shapes of the biplane fluoroscopic images A-B using a projection matrix.

The error for each of the 16 tested shapes is summarized visually in Figure 8. Table I summarises the mean and std error of the different $3 \mathrm{D}$ reconstruction performance.

TABLE I

COMPARATIVE TABLE DEPICTING THE SHAPE RECONSTRUCTION ACCURACY OF THE 3 DIFFERENT RECONSTRUCTED SHAPES COMPARED TO THE GROUND TRUTH. THE RESULTS SUMMARIZE THE MEAN AND STD

\begin{tabular}{ccccccc}
\multicolumn{7}{c}{ ERROR OF THE 4 SHAPES. } \\
\hline Unit [mm] & Shape & 1 & 2 & 3 & 4 & all shapes \\
\hline Fluoroscopy & mean & 1.62 & 1.29 & 1.48 & 1.67 & 1.51 \\
& std & 0.71 & 0.6 & 0.65 & 0.61 & 0.04 \\
FBG & mean & 1.75 & 1.24 & 2.09 & 2.03 & 1.77 \\
& std & 0.67 & 0.48 & 0.74 & 1.17 & 0.29 \\
Fusion & mean & 1.5 & 1.15 & 1.54 & 1.69 & 1.47 \\
& std & 0.53 & 0.42 & 0.59 & 0.78 & 0.15 \\
\hline
\end{tabular}

The results show that for all the methods the shape reconstruction error is below $2 \mathrm{~mm}$. This is a promising result, especially considering that in medical applications, and cardiovascular applications in particular, accuracies in the order of $2 \mathrm{~mm}$ are expected [27]. The different results show that all 3 reconstruction methods allow achieving targeted performance. Fluoroscopic imaging showed to outperform the FBG estimation quality. However, this difference is limited to $0.26 \mathrm{~mm}$. The limitation to distinguish strain from twist with strain from bending (for FBG shape sensing) is taken into account with the fusion. Overall, Figure 8 shows that the proposed fusion method provides better results in the large majority of experiments both compared to FBG shape and fluoroscopy-based shape estimation. One can notice that the error in shape 2 is lower than the other shapes. When observing Figure 8 , it becomes clear that lower error is not only due to shape 2 but also apparent in other shape. It likely depends on relative pose of a shape with respect to the fluoroscopy. This could imply a presence of uncertainty on the localisation of the landmarks in some poses than in others and that by accident shape 2 was placed in well conditioned poses in all 4 tests. Furthermore, a study of curvature and torsion impact on the accuracy of the reconstruction was performed. This test showed better reconstruction when accurate estimation of curvature and torsion. 

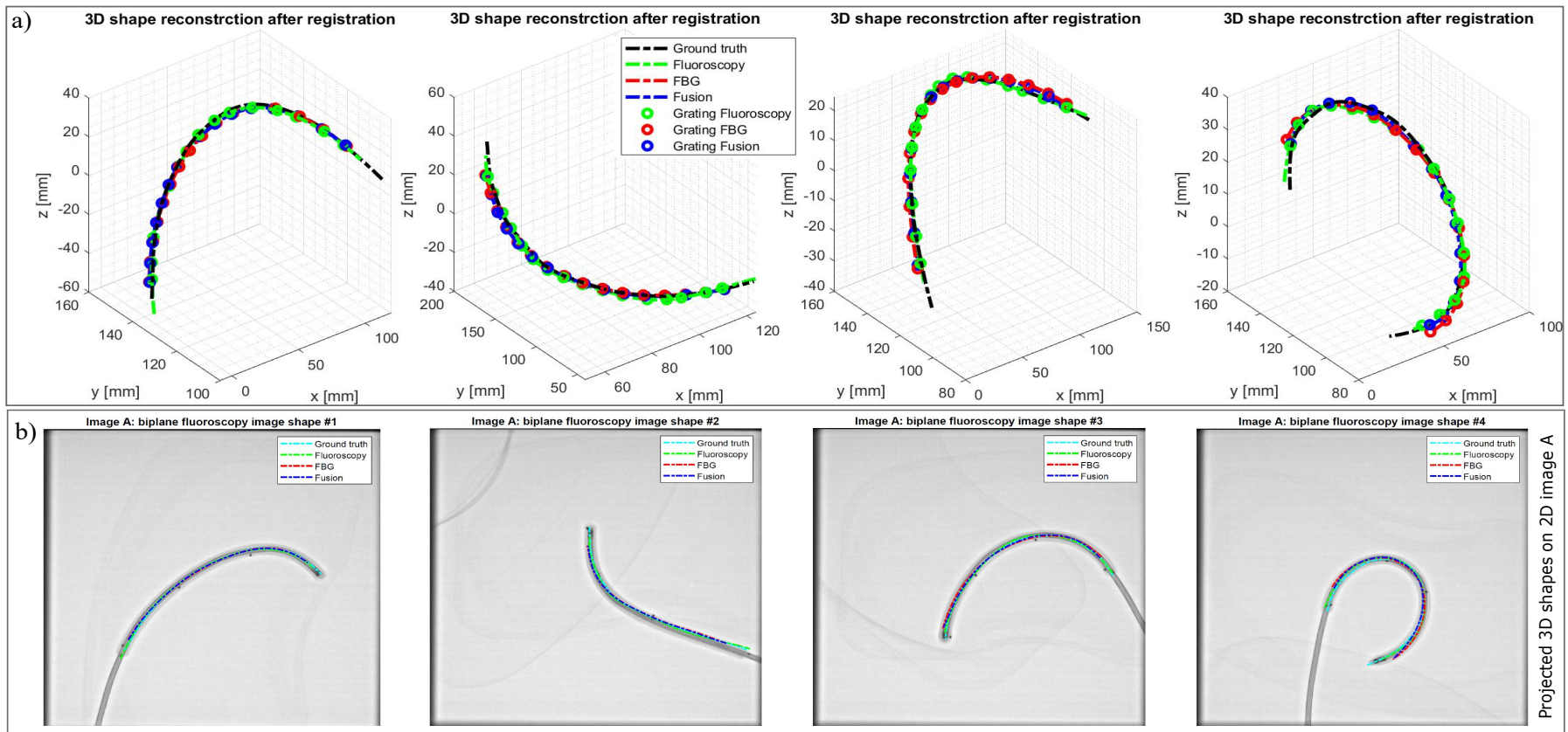

$\begin{array}{llll}y[\mathrm{~mm}] & 80 & 0 & \times[\mathrm{mm}]\end{array}$
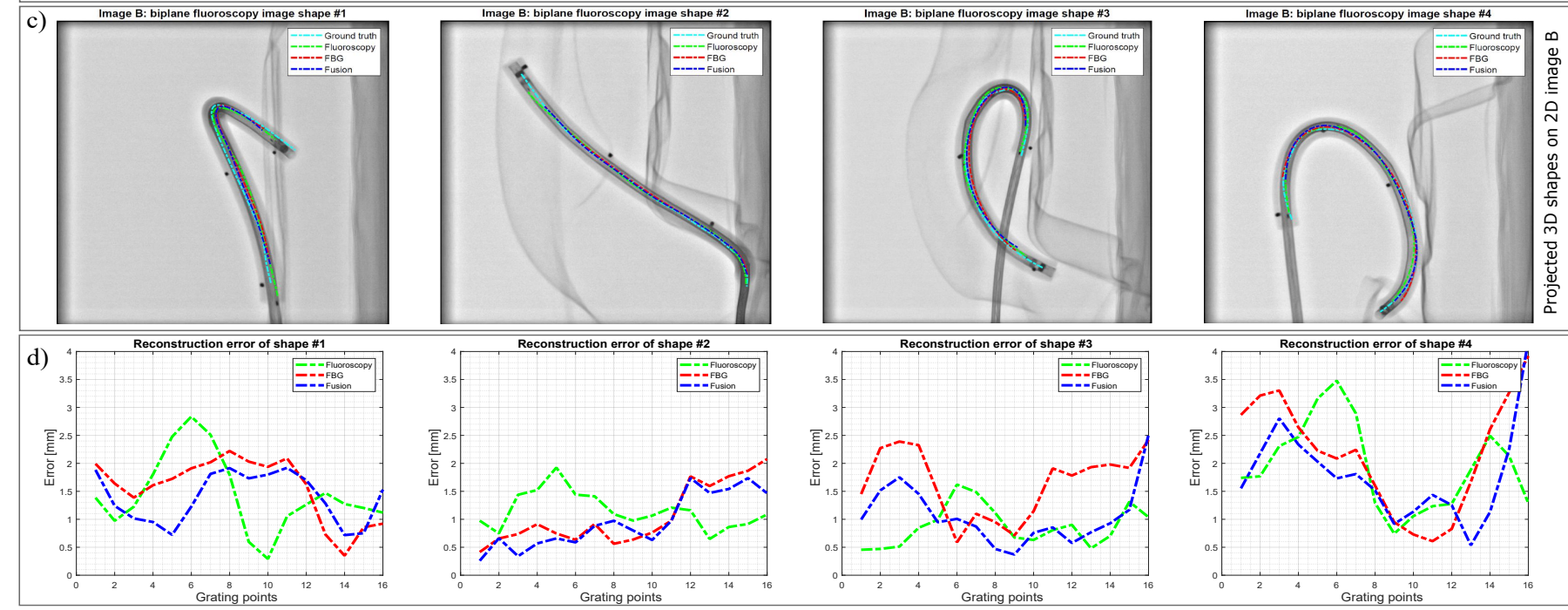

Fig. 6. Result of 4 reconstructed shapes in the absolute coordinate frame $O_{f}$. The 4 depicted shapes are selected randomly from the 4 performed test for each shape with different position and orientation. The used shapes are: ground truth, fluoroscopy, FBG and fusion corresponding to CT center line, biplane fluoroscopy, rigid fusion and dynamic fusion approach, respectively. a) 3D reconstructed shape, b) Projection on the the first biplane image (A), b) Projection on the the second biplane image (B) and c) distance between each of the shapes: ground truth vs.FBG, biplane fluoroscopy and fusion.

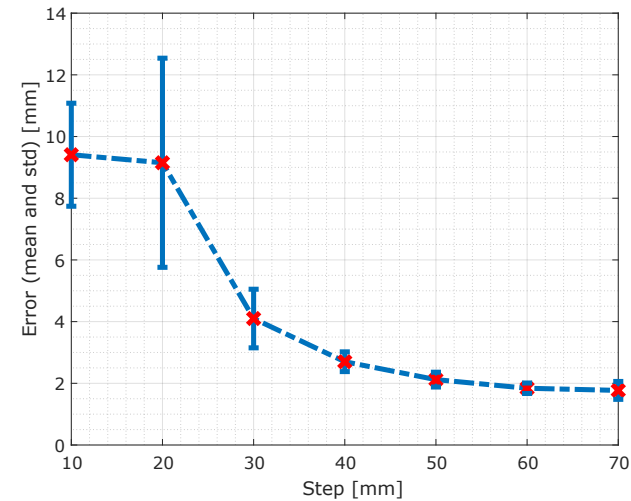

Fig. 7. Mean and standard deviation error measured between the FBG in $O_{f}$ frame for different markers step changes.

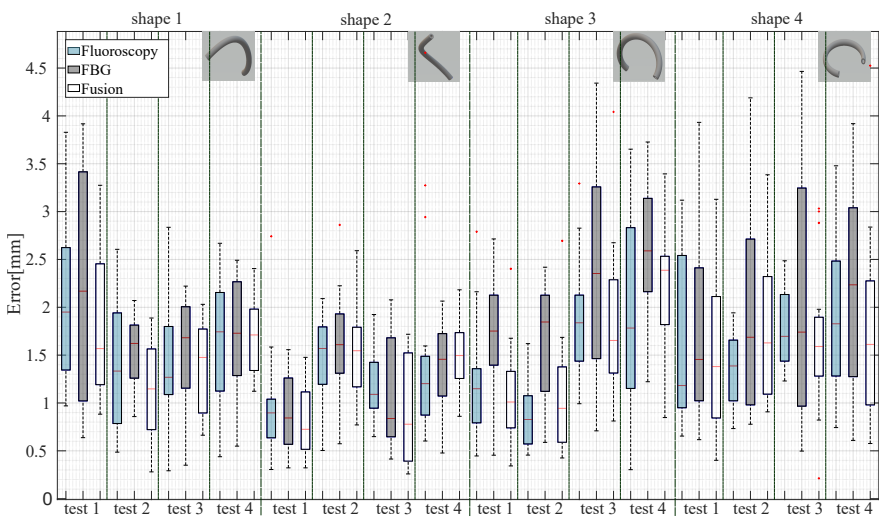

Fig. 8. 3D shape reconstructed error using three reconstruction methods with respect to the ground truth (where Fluoroscopy is the biplane approach, FBG is the rigid fusion and fusion is the dynamic fusion approach). 


\section{CONCLUSIONS}

This paper proposes and evaluates 3 different catheter shape reconstruction methods. Biplane fluoroscopy was compared to FBG shape and fusion of the both sensing reconstruction. An experimental setup was built hereto and tests were performed in the CathLab. To provide a reliable analysis, 4 different 3D shapes were considered during the experiment. Furthermore, each of the 4 shapes was tested at different poses with respect to an orthogonal biplane fluoroscopy setup. The 3 proposed methods for shape reconstruction showed satisfying performance comparatively to the expected error in such application with a mean error less than $2 \mathrm{~mm}$.

Until now, most papers (e.g. [10]) that explored FBG shape reconstruction performed an analysis in a coordinate system linked to the catheter. This means that these results did not include the error that originates from a rigid transformation that is necessary to represent this shape in a fixed absolute coordinate frame. As many practical uses of FBG shape reconstruction will require such absolute reference frame this error should be counted for as is done in this work. Further, we explored two methods for shape reconstruction that make use of FBG shape reconstruction. The conducted experiments demonstrate that fusion based approaches can offer comparable results to fluoroscopy reconstructions and show a small advantage of $0.3 \mathrm{~mm}$ of the dynamic fusion approach over the rigid fusion. Despite these promising result, practical application is limited to solutions that can warrant non-colinearity of the 3 corresponding markers.

The proposed fusion approaches can be readily implemented for catheter localization as their accuracy compares well to both CBCT and fluoroscopy reconstruction. Furthermore, opting for FBG-fluoroscopy fusion may substantially reduce the radiation dose that is typically associated with mere fluoroscopic shape reconstruction as it would allow lowering the frame-rate of the fluoroscope or allow asynchronous fluoroscopy only at instants where there is a large uncertainty or where the catheter approaches critical structures. Future work will investigate how these methods can provide automatic and real time 3D information for visual or robotic guidance towards safer navigation of the catheter. Another direction is to evaluate the real time performance of the fusion and to what extent the FBG will lower the x-ray exposure.

\section{ACKNOWLEDGMENT}

This work was supported by SF-REACTA project (3E180412) as well as Flemish Agency of Innovation and Entrepreneurship (VLAIO - HBC.2018.2046) and by ATLAS project. This project has received funding from the European Union's Horizon 2020 research and innovation programme under the Marie Sklodowska-Curie grant agreement No. 813782.

\section{REFERENCES}

[1] V. Vitiello, S.-L. Lee, and et al., "Emerging robotic platforms for minimally invasive surgery," IEEE reviews in biomedical engineering, vol. 6, pp. 111-126, December 2012.

[2] A. Ali, D. H. Plettenburg, and P. Breedveld, "Steerable catheters in cardiology: Classifying steerability and assessing future challenges," IEEE Transactions on Biomedical Engineering, vol. 63, no. 4, pp. 679693, 2016.
[3] J. M. Tobis and I. Abudayyeh, "New devices and technology in interventional cardiology," Journal of cardiology, vol. 65 , no. 1, pp. 5-16, 2015.

[4] T. Alderliesten, M. K. Konings, and W. J. Niessen, "Modeling friction, intrinsic curvature, and rotation of guide wires for simulation of minimally invasive vascular interventions," IEEE Transactions on Biomedical Engineering, vol. 54, no. 1, pp. 29-38, 2006.

[5] W. Tang, P. Lagadec, and et al., "A realistic elastic rod model for realtime simulation of minimally invasive vascular interventions," The Visual Computer, vol. 26, no. 9, pp. 1157-1165, 2010.

[6] M. Wagner, S. Schafer, and et al., "4d interventional device reconstruction from biplane fluoroscopy," Medical physics, vol. 43, no. 3, pp. 1324-1334, 2016.

[7] A. M. Franz, T. Haidegger, and et al., "Electromagnetic tracking in medicine-a review of technology, validation, and applications," IEEE transactions on medical imaging, vol. 33, no. 8, pp. 1702-1725, 2014.

[8] R. Chan, R. a. Manzke, and et al., "Optical shape sensing fiber for tip and shape characterization of medical instruments," U.S. Patent No. $9,693,707.4$ Jul. 2017

[9] P. T. Tran, P.-L. Chang, and et al., "3d catheter shape reconstruction using electromagnetic and image sensors," Journal of Medical Robotics Research, vol. 2, no. 03, pp. 1-13, 2017.

[10] O. Al-Ahmad, M. Ourak, and et al., "Improved fbg-based shape sensing methods for vascular catheterization treatment," IEEE Robotics and Automation Letters, vol. 5, no. 3, pp. 4687-4694, 2020.

[11] Q. Qiao, D. Willems, and et al., "Estimating and localizing external forces applied on flexible instruments by shape sensing," in 19th International Conference on Advanced Robotics. IEEE, 2019, pp. 227233.

[12] D. L. Presti, C. Massaroni, and et al., "Fiber bragg gratings for medical applications and future challenges: A review," IEEE Access, vol. 8, pp. $156863-156888,2020$

[13] K. R. Henken, J. Dankelman, and et al., "Error analysis of fbg-based shape sensors for medical needle tracking," IEEE/ASME Transactions on mechatronics, vol. 19, no. 5, pp. 1523-1531, 2013.

[14] F. Khan, A. Denasi, D. Barrera, and et al., "Multi-core optical fibers with bragg gratings as shape sensor for flexible medical instruments," IEEE sensors journal, vol. 19, no. 14, pp. 5878-5884, 2019.

[15] R. Xu, A. Yurkewich, and R. V. Patel, "Curvature, torsion, and force sensing in continuum robots using helically wrapped fbg sensors," IEEE Robotics and Automation Letters, vol. 1, no. 2, pp. 1052-1059, 2016.

[16] X. Ha, M. Ourak, O. Al-Ahmad, and et al., "Robust catheter tracking by fusing electromagnetic tracking, fiber bragg grating and sparse fluoroscopic images," TechRxiv, pp. 1-12, 2021.

[17] S. De Buck, F. Maes, and el al., "An augmented reality system for patient-specific guidance of cardiac catheter ablation procedures," IEEE Transactions on Medical Imaging, vol. 24, no. 11, pp. 1512-1524, 2005.

[18] S. De Buck, J. Ector, and et al., "Toward image-based catheter tip tracking for treatment of atrial fibrillation," in CI2BM09-MICCAI Workshop on Cardiovascular Interventional Imaging and Biophysical Modelling, pp. 1-8, 2009.

[19] Y. Ma, N. Gogin, and et al., "Real-time x-ray fluoroscopy-based catheter detection and tracking for cardiac electrophysiology interventions," Medical physics, vol. 40, no. 7, pp. 071902-071915, 2013.

[20] J. P. Moore and M. D. Rogge, "Shape sensing using multi-core fiber optic cable and parametric curve solutions," Optics express, vol. 20, no. 3, pp. 2967-2973, 2012.

[21] T. F. Banchoff and S. T. Lovett, Differential geometry of curves and surfaces. CRC Press, 2016.

[22] D. Carroll, E. Köse, and I. Sterling, "Improving frenet's frame using bishop's frame," arXiv:1311.5857, pp. 1-11, 2013.

[23] F. Khan, A. Donder, and et al., "Pose measurement of flexible medical instruments using fiber bragg gratings in multi-core fiber," IEEE Sensors Journal, vol. 20, no. 18, pp. 10955-10962, 2020.

[24] K. S. Arun, T. S. Huang, and S. D. Blostein, "Least-squares fitting of two 3-d point sets," IEEE Transactions on pattern analysis and machine intelligence, no. 5, pp. 698-700, 1987.

[25] A. F. Frangi, W. J. Niessen, and et al., "Multiscale vessel enhancement filtering," in International conference on medical image computing and computer-assisted intervention. Springer, 1998, pp. 130-137.

[26] P.-L. Chang, A. Rolls, and et al., "Robust catheter and guidewire tracking using b-spline tube model and pixel-wise posteriors," IEEE Robotics and Automation Letters, vol. 1, no. 1, pp. 303-308, 2016.

[27] N. M. De Groot, M. Bootsma, and et al., "Three-dimensional catheter positioning during radiofrequency ablation in patients: First application of a real-time position management system," Journal of cardiovascular electrophysiology, vol. 11, no. 11, pp. 1183-1192, 2000. 